\title{
Teaching of geometry in Bulgaria
}

\author{
Kiril Bankov \\ Faculty of Mathematics and Informatics, University of Sofia \\ For correspondence: kbankov@fmi.uni-sofia.bg
}

\begin{abstract}
:
Geometry plays an important role in the school mathematics curriculum all around the world. Teaching of geometry varies a lot (Hoyls, Foxman, \& Kuchemann, 2001). Many countries revise the objectives, the content, and the approaches to the geometry in school. Studies of the processes show that there are not common trends of these changes (Mammana \& Villani, 1998). This paper describes the recent state of geometry teaching in Bulgaria. The main ideas and principles in the development of school geometry are presented. The preparation of future mathematics teachers to teach the subject is also considered.
\end{abstract}

Keywords: geometry curriculum, axiomatic system, teacher preparation, argumentation, formal proof.

Geometry arose as a practical activity helping in measuring of heights and distances in the early civilizations. It was also used in elementary astronomy and the building of temples in ancient cultures (Mesopotamian, Egyptian, Indian, etc.). This practical knowledge was adapted by the Greek culture that developed geometry as an intellectual activity from around 600BC. The main feature of this period that stretches from Thales (640BC) to Diophantus (250AD) is the attempt to understand the world by rational arguments. The Euclidean approach to geometry in "Elements" (Euclid's Elements of Geometry, 2008) became the model for presenting mathematical ideas for centuries. Geometry has been an important part of many cultures and an incentive to the development of numerous mathematical theories.

Geometry is relevant and important in the modern mathematics and science. It lies in the heart of both theoretical and applied mathematics. Geometry is fundamental to physics and many science brunches are pervaded by geometrical aspects. There is no doubt in the usefulness of geometry in architecture (Sterk, 2008), technology (McDougall, McGeehe, 2001) and almost the whole everyday world (http://www.studymode.com/essays/Geometry-In-Everyday-Life-408843.html).

This is why geometry has been, and still is, an important part of the mathematics curriculum for many years. The objectives in teaching geometry differ across educational systems, but it seems that the following are common and the most important:

a) to build up knowledge of geometrical plane figures and solids and to use their properties to understand facts and to reason geometrically;

b) to solve practical problems by using geometry inside and outside mathematics;

c) to help in the understanding of how geometry describes objects and how the objects can be build;

d) to create the understanding of the importance of argumentation; from acceptable simple explanations towards more precise arguments and formal abstract proofs.

In fact, the last object is controversial. There is no doubt that Euclidean geometry is the area of mathematics in which proofs are first encountered in formal way. It is also true that for some pupils 
geometry is a suitable area to begin presenting proofs. Although the understanding and constructing a formal proof is important in geometry and in school mathematics as a whole, teaching and learning about this is not an easy and a straightforward process (Jones \& Rodd, 2001). The teachers need a lot of time and patience as well as trained skills to do this. It should also be accepted that not all pupils can reach the level to produce formal proofs.

Probably because of the last arguments, and also because pupils find geometry a difficult area, there has been a decline in teaching theoretical knowledge in geometry curriculum in many countries (Whiteley, 1999). What usually is taught in geometry classes concerns the knowledge and skills needed to solve standard geometrical problems, most of which require only simple computational procedures.

It should be acknowledged that for many reasons there is no way back to the "old way" of studying geometry in school as an academic discipline. But every effort should be done to keep the above mentioned valuable principal objectives in teaching geometry in school (http://njwildberger.wordpress.com/2012/08/03/curriculum-issues-and-geometry-in-education-40/).

Even though they are not clearly worded in the official curriculum, these are the main objectives in teaching geometry in Bulgarian school (Bulgarian School Curriculum and Educational Program). As in most places, instead of having objectives in teaching geometry, in Bulgarian curriculum there are statements on the approach to school geometry, a list of topics that should be covered, and vague explanations about the need of argumentation in geometry. This does not mean neither that the school geometry in Bulgaria consists of a set of not very well connected topics, nor that it deeply studies one or two particular topics. In order to better understand the position of geometry in Bulgarian school, a brief explanation of the educational system is presented below.

Bulgaria has three education levels: pre-school ( 3 to 6 or 7 years old), school education, and higher education. School education consists of basic and secondary education. Basic education includes primary school (Grades 1 to 4 ) and pre-secondary or middle school (Grades 5 to 8 ). Pupils enter basic education after they complete age 6 (in many cases they are about 7 years old). Education is compulsory up to the age 16 .

Secondary education is divided into general and vocational. Secondary general education can be obtained at secondary comprehensive schools (4 years) or profile-oriented schools (4 or 5 years). Students can enter the profile-oriented schools (mainly focused on mathematics and foreign languages) beginning in Grade 8 (rarely in Grade 9) after passing two entrance examinations: one in Bulgarian language and literature and one in mathematics or another subject according to the school's profile. Most of these schools offer intensive foreign language instruction.

Secondary vocational education can be obtained at technical schools beginning in Grade 9 for a fouryear training period or in Grade 8 for duration of five years. Professional technical schools also provide intensive foreign language instruction. The professional qualification obtained provides access to the labor market.

The geometric curriculum in basic and a part of pre-secondary education, till the end of Grade 6, is not systematic (Bulgarian School Curriculum and Educational Program). It is based on the realistic or practical approach to geometry. This means that the visualization and practical activities with real objects help pupils understand the world. The geometrical figures are considered as dynamical objects and can be used for visual representation of geometrical relations. The idea is that the sensible obtained information can be (later on) easily put in theoretical and abstract frames. 
The systematic and theoretical studying of geometry begins in Grade 7 (Table 1). Features of this development are:

a) The approach is a mixture of traditional Euclidean geometry and a small portion of transformational geometry with obvious emphasis on the former. Axiomatic introduction to geometry is used. Geometrical transformations are additional tools for argumentation, proof and rarely for solving problems.

b) There is a strong emphasis on congruence and similarity but they are taught separately, namely congruence in Grade 7, similarity in Grade 9. The philosophy under this is that congruence and similarity represent different levels of abstraction in geometry. The former considers congruent figures as "undistinguishable", while the latter makes the same with similar figures.

c) Pythagorean Theorem is introduced relatively late, in Grade 9. This is because to make use of this theorem (not only working with Pythagorean triples) pupils need to know the notion of irrational numbers (they need a square root). In algebra curriculum in Bulgaria irrational numbers are introduced in Grade 8 and studied in Grade 9.

d) Studying of plane geometry finishes in Grade 10. In Grade 11 pupils study trigonometry. In Grade 12 they study 3D geometry.

Table 1. Content of geometry curriculum in Bulgaria (Grades from 7 till 12)

\begin{tabular}{|c|c|}
\hline Grade & Topics \\
\hline Grade 7 & $\begin{array}{l}\text { Axioms in geometry } \\
\text { Basic geometrical figures and their elements (segment, angle, triangle, perpendicular } \\
\text { bisector, angle bisector, median, altitude, etc.) } \\
\text { Parallel lines and properties of the angles when intersecting them } \\
\text { Sum of the angles in a triangle } \\
\text { Congruent triangles } \\
\text { Inequality between the sides and the angles in a triangle } \\
\text { Quadrangle, parallelogram, trapezium. }\end{array}$ \\
\hline Grade 8 & $\begin{array}{l}\text { Vectors } \\
\text { Center of gravity of a triangle } \\
\text { Isometries (line and centre symmetry, rotation, translation) } \\
\text { Circle } \\
\text { Angles connected with a circle } \\
\text { Inscribed polygon, inscribed circle } \\
\text { Orthocentre of a triangle }\end{array}$ \\
\hline Grade 9 & $\begin{array}{l}\text { Proportional segments } \\
\text { Thales theorem } \\
\text { Similar triangles } \\
\text { Right angled triangle. Pythagoras } \\
\text { Trigonometric function in a right angled triangle }\end{array}$ \\
\hline Grade 10 & $\begin{array}{l}\text { Trigonometric functions in }\left[0^{\circ}, 180^{\circ}\right] \\
\text { Sine and Cosine theorems } \\
\text { Area of a polygon and a circle }\end{array}$ \\
\hline Grade 11 & Trigonometry \\
\hline Grade 12 & $\begin{array}{l}\text { 3-D geometry } \\
\text { Lines and planes in the space } \\
\text { Parallelepiped, pyramid, cylinder, cone }\end{array}$ \\
\hline
\end{tabular}

Traditionally, in Bulgaria the axiomatic approach to school geometry is used. The idea is developed by Euclid in "Elements" (Euclid's Elements of Geometry, 2008) and used as the only sensible 
approach to geometry for centuries. By the end of 19-th century several mathematicians elaborated the axiomatization of geometry. The most important work is done by David Hilbert in "Foundations of Geometry" (Hilbert, 1950). Later on mathematicians started using different sets of axioms leading to one and the same mathematical theory named as "Euclidean Geometry". Sets of axioms were developed especially suitable for introducing the axiomatic approach in school.

It should be acknowledged that it is not very appropriate to use a strict axiomatic approach for school pupils. The reasons are mostly didactical and pedagogical (Barnard, 2002). The approach in the Bulgarian curriculum is close to what other countries using axiomatic do, namely: a set of "agreed facts" is presented to the pupils as a set of axioms. This set contains part of the axioms but may also include some other facts. Then geometry is deductively built on these facts. Logically, this is the axiomatic approach but using not the complete and the minimal set of axioms.

In the official school documentation in Bulgaria (Bulgarian School Curriculum and Educational Program) there is not an explanation about the degree of argumentation that pupils in different school levels should reach. By the end of the last century Bulgaria was know as a country with strong emphasis in teaching argumentation and formal proofs, especially in geometry. Even if this is not any longer the case, there are still training in argumentation and proofs in geometry. The understanding is that since the teacher plays the main role in such an activity, it is important to give the teachers a relevant training and to make their pedagogical tasks as clear as possible. This especially concerns teachers in grades from 7 to 12 .

In Bulgaria, beginning in Grade 5, mathematics is taught by a teacher specifically prepared to teach mathematics. Typically, one mathematics teacher instructs the same cohort of students for the entire middle school education (from Grade 5 to Grade 8). Similarly, one mathematics teacher teaches all students in Grades 9 to Grade 12. In some schools, the same teacher instructs all students from Grade 5 to the end of Grade 12. Teacher preparation in Bulgaria is subject-matter oriented (Bankov, 2007). Future teachers receive their preparation in departments of mathematics. Courses in general pedagogy and psychology are taught by lecturers from departments of education. This means that teacher training on geometry, including the argumentation and proof, is a responsibility of mathematicians and mathematics educators.

For the preparation of mathematics teachers in this area there is usually a course called "School geometry". In the department of mathematics education at the University of Sofia this is a course of duration: 45 academic hours lectures (theory) and 60 academic hours exercise (using different methods for solving geometrical problems). (One academic hour is 45 minutes.) The book (Bankov \& Vitanov, 2003) is especially written for the purpose of this course. The main ideas and the content of the course are described below.

It starts with the presentation of a complete and minimal set of axioms for Euclidean geometry. Among the variety of such sets, the one developed by the Russian mathematician Kolmogorov (Egorov, 1979) especially for the purposes of the school education is chosen (Table 2). One of the features of this set is that the distance is a basic (postulated) notion. This is a useful advantage, since if the set of axioms does not postulate the distance it needs a lot of effort to build this notion. To make use of vectors and also to acquaint students with a different set of axioms the so called axiomatic of Weil is presented. The advantage of Weil's set of axioms is that it can easily be transformed to an axiomatic of three- and any other finite dimensional Euclidean geometry. 
Table 2. Set of axioms of Euclidean geometry (Kolmogorov)

\begin{tabular}{|c|c|}
\hline \multicolumn{2}{|c|}{ The basic sets are: the set of points and the set of lines. The plane is the set of all points. } \\
\hline Groups & Axioms \\
\hline Axioms of belonging. & $\begin{array}{l}\text { 1. Any line is a set of points. } \\
\text { 2. Any two different points determine only one line. } \\
\text { 3. There is at least one line and any line contains at least one point. }\end{array}$ \\
\hline Axioms of distance. & $\begin{array}{l}\text { 4. For any two points } A \text { and } B \text { a non-negative number } A B \text { is assign, which } \\
\text { is called a distance between } A \text { and } B . A B=0 \text { if and only if } A=B \text {. } \\
\text { 5. For any two points } A \text { and } B, A B=B A \text {. } \\
\text { 6. For any three points } A, B \text {, and } C, A C \leq A B+B C\end{array}$ \\
\hline \multicolumn{2}{|c|}{ Point $B$ is between points $A$ and $C$ if these three points are different and $A C=A B+B C$. } \\
\hline \multicolumn{2}{|c|}{$\begin{array}{l}\text { Let } \mathrm{A} \text { and } \mathrm{B} \text { are different points. Segment with end points } A \text { and } B \text { is the set of all points that are } \\
\text { between } A \text { and } B \text {. }\end{array}$} \\
\hline Axioms of order. & $\begin{array}{l}\text { 7. Let } p \text { is a line. Any point } O \text { from } p \text { partitions the points of } p \text { into two } \\
\text { non-empty sets in such a way that if } A \text { is from one of the sets and } B \text { is } \\
\text { from the other set, } O \text { is between } A \text { and } B \text {. Any of these sets is called a } \\
\text { ray with origin } O \text {. } \\
\text { 8. For any ray with origin } O \text { and for any non-negative number } a \text { there is a } \\
\text { unique point } A \text { on the ray such that } O A=a \text {. } \\
\text { 9. Three different points lie on a line if and only if one of them is between } \\
\text { the other two. } \\
\text { 10. Any line } p \text { partitions the points of the plane into two non-empty sets in } \\
\text { such a way that if } A \text { is from one of the sets and } B \text { is from the other set, } \\
\text { the segment } A B \text { and the line } p \text { have a common point. Any of these sets } \\
\text { is called a semi-plane with boundary } p \text {. }\end{array}$ \\
\hline \multicolumn{2}{|c|}{ Two lines are parallel if and only if they do not have a common point. } \\
\hline Axiom of parallel. & $\begin{array}{l}\text { 11. Let } M \text { is a point that does not belong to the line } p \text {. There is not more } \\
\text { than one line containing } M \text { and parallel to } p \text {. }\end{array}$ \\
\hline \multicolumn{2}{|c|}{$\begin{array}{l}\text { A one-to-one and onto mapping of a plane to the plane is called a transformation of the plane. A } \\
\text { transformation that preserves distance is called an isometry. }\end{array}$} \\
\hline Axiom of isometry. & $\begin{array}{l}\text { 12. Let } A \text { and } B \text { are different points, and } A_{1} \text { and } B_{1} \text { are such points that } \\
A_{1} B_{1}=A B \text {.Then there are exactly two isometries } f \text { and } g \text {, that map } A \text { into } \\
A_{1} \text { and } B \text { into } B_{1} \text {. If } \alpha \text { is one of the semi-planes with boundary } A B, \beta \text { and } \\
\gamma \text { are the two semi-planes with boundary } A_{1} B_{1} \text {, then } f \text { maps } \alpha \text { into } \beta \text {, and } \\
g \text { maps } \alpha \text { into } \gamma \text {. }\end{array}$ \\
\hline
\end{tabular}

Another useful feature of the Kolmogorov set of axioms is that the axiom of isometry connects the traditional Euclidean geometry and transformational geometry. This also gives a good starting point for teaching of isometries in the plane. It ends with the theorem for the classification of the congruencies that describes all isometries in the plane. The next set of transformations is similarities. As in the school geometry curriculum, they are studied after the isometries for the same reason. The end point is again the theorem for the classification of the similarity transformations. Affinity in geometry as a special kind of transformation is also considered.

Special attention is paid to the geometrical definition of the area of a polygon. The main result is the theorems for existence and for the uniqueness of the area. Next follows the geometry of circles. It includes inversion, radical axis, and coaxial systems of circles. The plane geometry ends with several classical theorems such as the theorems of Ceva and Menelaus, the nine point circle and the theorem 
of Feuerbach, the theorem of Miquel and Simson lines (Johnson, 1960). In the school curriculum these theorems are taught only to students in elective classes of mathematics.

The spatial geometry is not systematically presented. Three topics are covered: trihedral angles (including cosine and sine theorems), the theorem of Euler for solids, and the Platonic solids. These topics are not covered in the ordinary school curriculum. Since small amount of time is left for the 3D geometry, the lecturers chose topics taught to students in elective classes of mathematics.

A substantial portion of the course is devoted to solving geometrical problems connected with the above mentioned topics. Part of the topics are taken from (or are very close to) geometric textbooks. Other problems are more conceptual or consider typical geometric situations. The time spent from future teachers on solving problems gives not only the training in specific geometrical techniques but also provide them with a rich set of problems that can be used later in their professional work in the school.

Turning back to the school curriculum, the recent studies show that pupils' achievement in geometry in Bulgaria is getting worse and worse. TIMSS results (Mullis, Martin, Foy, 2008) show that in geometry area Bulgarian 8-th grade students perform significantly lower than the TIMSS scale average. Unfortunately, there are not much data from other studies of students' mathematics achievement in Bulgaria. Nevertheless, for many teachers and mathematicians it is clear that pupils may (more or less) solve geometrical problems that are algorithmic or involve straightforward calculations. But most school students cannot deal with problems that need (even a small portion of) imagination, creativity, conjecturing, or argumentation.

Results of three geometrical items taken from the national mathematics assessment in grade 7 in Bulgaria in May 2010 (the end of the school year) are presented below. These are constructed response items. The correct response is marked with a + sign. For each option the percent of responses are given in the brackets.

The figure shows three straight lines intersecting in one point. What is the degree measure of angle $\alpha$ ?

A) $50^{\circ}(16.1 \%)$

+B) $80^{\circ}(55.5 \%)$

C) $90^{\circ}(10.6 \%)$

D) $100^{\circ}(17.0 \%)$

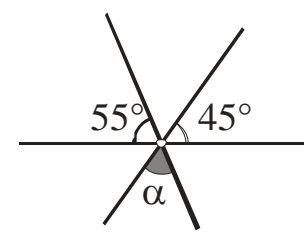

(Omit: 0.8\%)

In the picture, $\square A B C$ is isosceles $(A C=B C)$. The perpendicular bisector of $B C$ intersects $A C$ in $M$. The perimeter of $\square A B M$ is $13 \mathrm{~cm}$, and $A B=4 \mathrm{~cm}$. Find the perimeter of $\square A B C$ :
A) $26 \mathrm{~cm}(24.8 \%)$
+B) $22 \mathrm{~cm}(28.8 \%)$
C) $21 \mathrm{~cm}(16.5 \%$
D) $17 \mathrm{~cm}(27.6 \%)$

(Omit: 2.3)

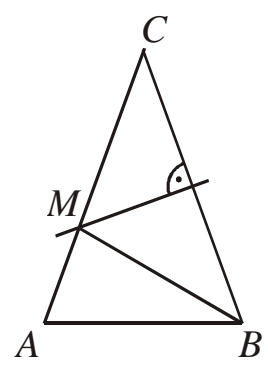


In the picture, $A O$ and $B O$ are angle bisectors in $\square A B C$. If $\angle A O B=120^{\circ}$ and $\angle O A C=25^{\circ}$, what is the degree measure of $\angle A B C$ :
A) $25^{\circ}(12.8 \%)$
B) $35^{\circ}(33.1 \%)$
C) $50^{\circ}(16.9 \%)$
+D) $70^{\circ}(35.4 \%)$

(Omit: $1.8 \%)$

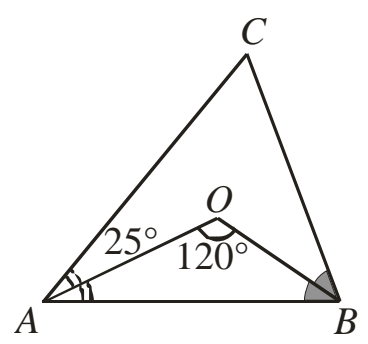

Many reasons may contribute to these not satisfying results, but two are worth mentioning: (i) the decline of geometrical knowledge in the school curriculum; (ii) the teachers are less and less prepared to teach geometry. The last reason may seem strange having in mind the above explanation of the preparation of the future teachers. What happens is that "School geometry" is a difficult study for the future mathematics teacher. They need to put a lot of work and effort to successfully complete the course. Many of them finish the study with mediocre knowledge and skills to teach geometry. The existence of "good" textbooks cannot diminish this effect. Teaching geometry is not only presenting geometrical facts and showing the solution of geometry problems. It needs imagination, methodological skills, and the most important, deep understanding of the ideas, methods and constructions in school geometry.

\section{References}

Bankov, K. (2007). The Influence of the World Educational Changes on the Teacher Education System in Bulgaria. In Reforming Teaching Globally, edited by M. T. Tatto, Oxford Studies in Comparative Education.

Bankov, K., \& Vitanov, T. (2003). Geometry. Anubis (in Bulgarian).

Barnard, T. (2002). Starting Points and End Points. Reasoning, explanation and proof in school mathematics and their place in the intended curriculum. Proceedings of the QCA international seminar, 4-6 October 2001, Cambridge, England. Qualifications and Curriculum Authority.

Bulgarian School Curriculum and Educational Program. http://www.mon.bg/top_menu/general/educational_programs/ (in Bulgarian).

Egorov, I. P. (1979). Geometry. Prosvjashtenie, Moscow (in Russian).

Euclid's Elements of Geometry. (2008). Edited and provided with modern English translation by Richard Fitzpatrick. http://farside.ph.utexas.edu/euclid/elements.pdf.

Hilbert, D. (1950). The Foundations of Geometry. Authorized translation by E. J. Townsend. http://www.gutenberg.org/files/17384/17384-pdf.pdf.

Hoyls, C., Foxman, D., \& Kuchemann, D. (2001). Comparative Studies in Geometry Curricula. Institute of Education, University of London: QCA.

Johnson, R. (1960). Advanced Euclidean Geometry. Dover Publications, INC.

Jones, K. \& Rodd, M. (2001). Geometry and Proof. British Society for Research into learning Mathematics. http://eprints.soton.ac.uk/14691/1/Jones_Rodd_BSRLM_21-1_2001.pdf

Mammana, C. \& Villani, V. (1998). Perspectives on Teaching of Geometry for the 21-st Century. Dortrecht: Kluwer.

McDougall, D. \& McGehee, J. (2001). Geometry and Technology. Proceedings of the annual meeting of PME. http://files.eric.ed.gov/fulltext/ED476613.pdf\#page=83

Mullis, I., Martin, M., \& Foy, P. (2008). TIMSS2007 International Mathematics Report. Boston College, IEA.

Sterk, H. (2008). Geometry in architecture and building. Faculteit Wiskunde en Informatica Technishe Universiteit Eindhoven. http://www.win.tue.nl/ sterk/Bouwkunde/hoofdstuk1.pdf

Whiteley, W. (1999). The Decline and Rise of Geometry in 20 ${ }^{\text {th }}$ Century North America. Proceedings of the 1999 CMESG Conference. http://www.math.yorku.ca/Who/Faculty/Whiteley/cmesg.pdf 